\title{
Graphene: from materials science to particle physics.
}

Joaquín E. Drut*†

Department of Physics, The Ohio State University, Columbus, OH 43210-1117, USA

E-mail: jdrut@mps.ohio-state.edu

\section{Timo A. Lähde}

Helsinki Institute of Physics and Department of Applied Physics, P. O. Box 14100, Aalto University, FI-00076 Aalto, Espoo, Finland

E-mail: timo.lahde@tkk.fi

\section{Eero Tölö}

Department of Applied Physics, P. O. Box 14100, Aalto University, FI-00076 Aalto, Espoo, Finland

E-mail: eero.tolo@tkk.fi

Since its discovery in 2004, graphene, a two-dimensional hexagonal carbon allotrope, has generated great interest and spurred research activity from materials science to particle physics and vice versa. In particular, graphene has been found to exhibit outstanding electronic and mechanical properties, as well as an unusual low-energy spectrum of Dirac quasiparticles giving rise to a fractional quantum Hall effect when freely suspended and immersed in a magnetic field. One of the most intriguing puzzles of graphene involves the low-temperature conductivity at zero density, a central issue in the design of graphene-based nanoelectronic components. While suspended graphene experiments have shown a trend reminiscent of semiconductors, with rising resistivity at low temperatures, most theories predict a constant or even decreasing resistivity. However, lattice field theory calculations have revealed that suspended graphene is at or near the critical coupling for excitonic gap formation due to strong Coulomb interactions, which suggests a simple and straightforward explanation for the experimental data. In this contribution we review the current status of the field with emphasis on the issue of gap formation, and outline recent progress and future points of contact between condensed matter physics and Lattice QCD.

The XXVIII International Symposium on Lattice Field Theory

June 14-19,2010

Villasimius, Sardinia, Italy

\footnotetext{
${ }^{*}$ Speaker.

†Present address: Theoretical Division, Los Alamos National Laboratory, Los Alamos, NM, 87545, USA.
} 


\section{Introduction}

The recent experimental isolation of single atomic layers of graphite, known as graphene, has provided physicists with a novel opportunity to study a system with remarkable electronic and many-body properties, which is easy to manipulate experimentally [1,2]. Even more recently, the advent of experiments utilizing samples of suspended graphene, free from the interference of an underlying substrate [3], has provided unprecedented insight into the intrinsic properties of graphene. Among other remarkable discoveries, suspended graphene has been found to exhibit a carrier mobility which exceeds that of silicon by an order of magnitude, a fractional quantum Hall effect which is indicative of strong electron-electron interactions, as well as a markedly nonmetallic behavior of the DC conductivity at low temperatures.

A central property of graphene is that the low-energy electronic spectrum can be described in terms of two flavors of massless, four-component fermionic quasiparticles with linear dispersion [4]. Indeed, due to the hexagonal honeycomb arrangement of the carbon atoms in the graphene lattice, the band structure of graphene exhibits two inequivalent (but degenerate) "Dirac cones" where the conduction and valence bands touch. Since the energy-momentum relation around a Dirac point is linear as in relativistic theories, the low-energy description of graphene bears a certain resemblance to massless Quantum Electrodynamics (QED). Nevertheless, an important difference is that the Fermi velocity of the quasiparticles in graphene is as low as $v_{F} \simeq c / 300$, whereby the electromagnetic interaction is rendered essentially instantaneous. However, it should be pointed out that such a value of $v_{F}$ is actually unusually large from a condensed matter point of view.

Such an unusual band structure (often referred to as semimetallic) accounts fairly well for the observed properties of graphene sheets deposited on a dielectric substrate. While suspended graphene has recently come under intense experimental investigation [3], its spectrum is yet to be computed in a controlled fashion. From a theoretical perspective, the challenging feature of suspended graphene lies in the smallness of the dieletric constant $\varepsilon=\varepsilon_{0}$ which, in conjunction with the small value of $v_{F}$, results in a graphene analogue of the fine-structure constant $\alpha_{g} \gtrsim 1$. At such strong coupling, a dynamical transition into a phase fundamentally different from the weaklycoupled semimetallic phase of graphene is a strong possibility. In graphene sheets deposited on a substrate, such a transition is effectively inhibited due to the suppression of $\alpha_{g}$ by the dielectric.

\section{Low-energy effective theory}

The electronic band structure of graphene close to the Fermi level forms the basis of the lowenergy effective theory of graphene. This band structure is a reflection of the hexagonal arrangement of the carbon atoms, which can be decomposed into two triangular sublattices $A$ and $B$. This leads to the tight-binding model

$$
H=-t \sum_{\langle i, j\rangle, \sigma=\uparrow, \downarrow}\left(a_{\sigma, i}^{\dagger} b_{\sigma, j}+\text { h.c. }\right)-t^{\prime} \sum_{\langle\langle i, j\rangle\rangle, \sigma=\uparrow, \downarrow}\left(a_{\sigma, i}^{\dagger} a_{\sigma, j}+b_{\sigma, i}^{\dagger} b_{\sigma, j}+\text { h.c. }\right),
$$

where the operators $a_{\sigma, i}^{\dagger}\left(a_{\sigma, i}\right)$ and $b_{\sigma, i}^{\dagger}\left(b_{\sigma, i}\right)$ create (annihilate) an electron of spin $\sigma$ at location $i$ on sublattices $A$ and $B$, respectively. The first term (involving $t$ ) takes into account nearest-neighbor interactions (as first done by Wallace in Ref. [5]), and the second term (involving $t^{\prime}$ ) the nextto-nearest neighbor ones. The hopping parameters that give an optimal fit to the experimentally 
determined band structure of graphene are $t \simeq 2.8 \mathrm{eV}$ and $t^{\prime} \simeq 0.1 \mathrm{eV}$ [6]. The tight-binding model with nearest-neighbor hopping can be generalized to a hexagonal Hubbard model by addition of an on-site Coulomb repulsion term, which has been recently studied [7] within a Quantum Monte Carlo approach.

We shall follow a somewhat different route based on an Effective Field Theory (EFT) treatment of graphene $[8,9]$, which has the advantage of describing the physics of graphene directly in terms of the relevant low-energy degrees of freedom, namely charged massless fermionic quasiparticles. The EFT description of graphene has an additional advantage as it allows for the direct study of effects due to the unscreened, long-range Coulomb interactions between the quasiparticles. In what follows, we shall formulate a continuum Lagrangian field theory valid at low momenta, much smaller than the inverse of the interatomic distance $\sim 1.42 \AA$.

\subsection{Continuum formulation}

The low-energy EFT of graphene may be derived from a tight-binding or Hubbard model description augmented by a long-range Coulomb interaction [9], yielding a theory of $N_{f}$ Dirac flavors interacting via an instantaneous Coulomb interaction. The action (in Euclidean spacetime) of this theory is

$$
S_{E}=-\sum_{a=1}^{N_{f}} \int d^{2} x d t \bar{\psi}_{a} D\left[A_{0}\right] \psi_{a}+\frac{1}{2 g^{2}} \int d^{3} x d t\left(\partial_{i} A_{0}\right)^{2},
$$

where $N_{f}=2$ for graphene monolayers, $g^{2}=e^{2} / \varepsilon_{0}$ for graphene in vacuum (suspended graphene), $\psi_{a}$ is a four-component Dirac field in (2+1) dimensions, $A_{0}$ is a Coulomb field in $(3+1)$ dimensions, and

$$
D\left[A_{0}\right]=\gamma_{0}\left(\partial_{0}+i A_{0}\right)+v_{F} \gamma_{i} \partial_{i}, \quad i=1,2
$$

where the Dirac matrices $\gamma_{\mu}$ satisfy the Euclidean Clifford algebra $\left\{\gamma_{\mu}, \gamma_{v}\right\}=2 \delta_{\mu \nu}$. The fourcomponent spinor structure accounts for quasiparticle excitations of sublattices $A$ and $B$ around the two Dirac points in the band structure [4,9]. The two Dirac points are identified with the two inequivalent representations (with opposite parity) of the Dirac matrices in $(2+1)$ dimensions. In graphene monolayers, $N_{f}=2$ owing to electronic spin, while $N_{f}=4$ is related to the case of two decoupled graphene layers, interacting solely via the Coulomb interaction. Consideration of arbitrary $N_{f}$ is also useful, given that an analytic treatment [10] is possible in the limit $N_{f} \rightarrow \infty$.

The strength of the Coulomb interaction is controlled by $\alpha_{g}=e^{2} /\left(4 \pi v_{F} \varepsilon_{0}\right)$, which is the graphene analogue of the fine-structure constant $\alpha \simeq 1 / 137$ of QED. It is straightforward to show that $\alpha_{g}$ is the only parameter, by rescaling according to

$$
t^{\prime}=v_{F} t, \quad A_{0}^{\prime}=A_{0} / v_{F} .
$$

The action (2.2) is invariant under spatially uniform gauge transformations (see Sec. 3.1). Notice that since the $A_{0}$ field is (3+1)-dimensional, a four-fermion Coulomb interaction of the form

$$
\frac{\bar{\psi}_{a}(x) \gamma_{0} \psi_{a}(x) \bar{\psi}_{b}\left(x^{\prime}\right) \gamma_{0} \psi_{b}\left(x^{\prime}\right)}{\left|\mathbf{x}-\mathbf{x}^{\prime}\right|}
$$

is recovered by integrating out $A_{0}$. Nevertheless, for our purposes the original form of the action (quadratic in the fermions) as given in Eq. (2.2) is preferable. 
A central property of the low-energy EFT is that Eq. (2.2) respects a global $\mathrm{U}\left(2 N_{f}\right)$ chiral symmetry under the transformations

$$
\psi_{a} \rightarrow \exp \left(i \Gamma_{j} \alpha_{j}\right) \psi_{a}
$$

where the matrices $\Gamma_{j}$ are the $\left(2 N_{f}\right)^{2}$ hermitian generators of $\mathrm{U}\left(2 N_{f}\right)$, such that for the case of graphene monolayers, the group is $\mathrm{U}(4)$. It should be noted that the choice of any particular representation for the $\Gamma_{j}$ is completely arbitrary and is not necessary for any calculational purpose, as all relevant information is provided by the Clifford algebra. However, the identification of the spinor degrees of freedom with any particular Dirac point and graphene sublattice is dependent on the chosen representation. This $\mathrm{U}(4)$ chiral symmetry, which strictly speaking is a flavor symmetry, can be spontaneously broken down to $\mathrm{U}(2) \times \mathrm{U}(2)$, in which case the excitonic condensate $\langle\bar{\psi} \psi\rangle$ acquires a non-vanishing value, signaling the formation of quasiparticle-hole bound states. The same group structure is obtained by adding to Eq. (2.2) a parity invariant (Dirac) mass term

$$
\int d^{2} x d t m_{0} \bar{\psi}_{a} \psi_{a}
$$

which breaks the symmetry explicitly. For the extended theory with $N_{f}$ flavors, the symmetry breaking pattern is $\mathrm{U}\left(2 N_{f}\right) \rightarrow \mathrm{U}\left(N_{f}\right) \times \mathrm{U}\left(N_{f}\right)$. Other symmetry breaking patterns, involving the possibilites of magnetic as well as Cooper-like pairing instabilities, have been investigated in Refs. $[9,11]$.

\subsection{Effective action and probability measure}

The partition function corresponding to Eq. (2.2) is given by

$$
Z=\int \mathscr{D} A_{0} \mathscr{D} \psi \mathscr{D} \bar{\psi} \exp \left(-S_{E}\left[\bar{\psi}_{a}, \psi_{a}, A_{0}\right]\right)
$$

where it is possible to integrate out the fermionic degrees of freedom, as $S_{E}$ is quadratic in the $\psi_{a}$. We thus obtain

$$
Z=\int \mathscr{D} A_{0} \exp \left(-S_{E}^{g}\left[A_{0}\right]\right) \operatorname{det}\left(D\left[A_{0}\right]\right)^{N_{f}}
$$

where

$$
S_{E}^{g}=\frac{1}{2 g^{2}} \int d^{3} x d t\left(\partial_{i} A_{0}\right)^{2}
$$

is the pure gauge part of the action. It is of central importance for the convergence of the Monte Carlo algorithm that the above determinant has a definite sign, independently of any particular configuration of the gauge field $A_{0}$. One way to establish this property is to proceed by writing $D\left[A_{0}\right]$ in the form

$$
D\left[A_{0}\right]=\left(\begin{array}{cc}
M\left[A_{0}\right] & 0 \\
0 & -M\left[A_{0}\right]
\end{array}\right)=\left(\begin{array}{cc}
M\left[A_{0}\right] & 0 \\
0 & M^{\dagger}\left[A_{0}\right]
\end{array}\right)
$$

where

$$
M\left[A_{0}\right]=\sigma_{0}\left(\partial_{0}+i A_{0}\right)+v_{F} \sigma_{i} \partial_{i}, \quad i=1,2,
$$

which entails a specific choice of Dirac $\gamma$-matrices. Furthermore, we note that $A_{0}$ is real, and that the Pauli matrices and the momentum operator are hermitian. The latter implies $\partial_{\mu}^{\dagger}=-\partial_{\mu}$, and therefore

$$
\operatorname{det}(D)=\operatorname{det}(M) \operatorname{det}\left(M^{\dagger}\right)=|\operatorname{det}(M)|^{2}>0
$$


While this property is not affected by the introduction of a parity invariant mass term such as Eq. (2.7), the positivity of $\operatorname{det}(D)$ breaks down in the presence of a chemical potential.

The fact that $\operatorname{det}(D)$ is positive definite allows for the definition of an effective action that is purely real, given by

$$
S_{\text {eff }}\left[A_{0}\right]=-N_{f} \ln \operatorname{det}\left(D\left[A_{0}\right]\right)+S_{E}^{g}\left[A_{0}\right],
$$

so that the partition function becomes

$$
Z=\int \mathscr{D} A_{0} \exp \left(-S_{\text {eff }}\left[A_{0}\right]\right)
$$

where $P\left[A_{0}\right]=\exp \left(-S_{\text {eff }}\left[A_{0}\right]\right)>0$ can be interpreted as a positive definite probability measure for a Monte Carlo calculation, as outlined in Section 3.

\subsection{Operator expectation values}

The expectation value of a given operator $O[\bar{\psi}, \psi]$ dependent on the fermion fields can be calculated by taking functional derivatives of the generating functional

$$
Z[\bar{\eta}, \eta]=\int \mathscr{D} A_{0} \mathscr{D} \psi \mathscr{D} \bar{\psi} \exp \left(-S_{E}\left[A_{0}, \bar{\psi}, \psi, \bar{\eta}, \eta\right]\right),
$$

where source terms have been added to the original action according to

$$
S_{E}\left[A_{0}, \bar{\psi}, \psi, \bar{\eta}, \eta\right]=S_{E}\left[A_{0}, \bar{\psi}, \psi\right]+\int d^{2} x d t(\bar{\psi} \eta+\text { h.c. }),
$$

such that the modified effective gauge action is a functional of $A_{0}$ as well as of the sources $\eta, \bar{\eta}$. It is again possible to integrate out the fermionic degrees of freedom and take functional derivatives with respect to the sources in the resulting expression

$$
Z[\bar{\eta}, \eta] \propto \int \mathscr{D A} A_{0} \exp \left(-S_{\mathrm{eff}}\left[A_{0}\right]\right) \exp \left(-\int d^{2} x d t \bar{\eta} D^{-1}\left[A_{0}\right] \eta\right),
$$

which makes it possible to obtain expectation values in terms of a path integral over $A_{0}$ only. While this procedure is completely general, it is possible to employ a slightly different approach in order to facilitate the computation of the chiral condensate and susceptibility.

The chiral condensate $\sigma$, which is the order parameter of the semimetal-insulator phase transition in graphene, is defined by

$$
\sigma \equiv\left\langle\bar{\psi}_{b} \psi_{b}\right\rangle,
$$

where the fermion fields are evaluated at the same space-time point. It is useful to note that the mass $m_{0}$ plays the rôle of a source, coupled to $\bar{\psi}_{b} \psi_{b}$. The expectation value of this operator can therefore be obtained by first differentiating the partition function with respect to $m_{0}$ and dividing by the volume, giving

$$
\sigma=\frac{1}{V Z} \int \mathscr{D} A_{0} \mathscr{D} \psi \mathscr{D} \bar{\psi} \int d^{2} x d t \bar{\psi}_{b}(x, t) \psi_{b}(x, t) \exp \left(-S_{E}\right)=\frac{1}{V} \frac{\partial \ln Z}{\partial m_{0}},
$$

where $\sigma$ has been defined as an average over the lattice volume occupied by the fermions. On the other hand, once the fermions have been integrated out, the derivative with respect to $m_{0}$ yields

$$
\sigma=\frac{1}{V Z} \int \mathscr{D} A_{0} \operatorname{Tr}\left(D^{-1}\left[A_{0}\right]\right) \exp \left(-S_{\mathrm{eff}}\left[A_{0}\right]\right)=\frac{1}{V}\left\langle\operatorname{Tr}\left(D^{-1}\left[A_{0}\right]\right)\right\rangle,
$$


where the identities

$$
\operatorname{det}(D[\lambda])=\exp \left(\operatorname{Tr}(\log (D[\lambda])), \quad \frac{\partial \operatorname{det}(D[\lambda])}{\partial \lambda}=\operatorname{det}(D[\lambda]) \operatorname{Tr}\left(D^{-1}[\lambda] \frac{\partial D}{\partial \lambda}\right),\right.
$$

have been used. The chiral susceptibility $\chi_{l}$ may be found by taking one more derivative with respect to $m_{0}$, giving

$$
\chi_{l} \equiv \frac{\partial \sigma}{\partial m_{0}}=\frac{1}{V}\left[\left\langle\operatorname{Tr}^{2}\left(D^{-1}\right)\right\rangle-\left\langle\operatorname{Tr}\left(D^{-2}\right)\right\rangle-\left\langle\operatorname{Tr}\left(D^{-1}\right)\right\rangle^{2}\right],
$$

which is expected to diverge at a second-order phase transition, and may also yield constraining information on the universal critical exponents of the transition.

\section{Graphene on the lattice}

In this section we formulate the lattice version of Eq. (2.2) following Refs. [12, 13]. It should be noted in this context that a closely related lattice model of the strong-coupling limit of graphene has been considered in Ref. [14]. We begin by discretizing the pure gauge sector, where the requirement of gauge invariance implies the use of "link variables" to represent the gauge degrees of freedom. The "staggered" discretization of the fermionic sector is then outlined, as it is the preferred choice to represent fermions with chiral symmetry at finite lattice spacing [15, 16]. Throughout this paper, the lattice spacing is set equal to unity, and thus all dimensionful quantities should be regarded as expressed in units of the lattice spacing.

\subsection{Gauge invariance and link variables}

The pure gauge part of the Euclidean action, Eq. (2.10), can be thought of as the non-relativistic limit of the Lorentz-invariant form $\frac{1}{4} F_{\mu \nu} F^{\mu v}$ where $F_{\mu v}=\partial_{\mu} A_{v}-\partial_{v} A_{\mu}$, such that

$$
F_{\mu v} F^{\mu v}=F_{0 j} F^{0 j}+F_{i j} F^{i j}+F_{i 0} F^{i 0}=2 F_{0 j} F^{0 j}=2\left(\partial_{j} A_{0}\right)^{2},
$$

where we have used $F_{i j}=0$ (no magnetic field) and $\partial_{0} A_{j}=0$ (no electric field induction by a magnetic field), valid in the non-relativistic limit $\left(v_{F} \ll c\right)$. Thus, for graphene the only non-vanishing contribution is the electric field $E_{j}=-\partial_{j} A_{0}$, which represents the instantaneous Coulomb interaction between the quasiparticles.

The gauge action (2.10) is invariant under the time-dependent, spatially uniform gauge transformations

$$
A_{0} \rightarrow A_{0}+\alpha(t), \quad \psi \rightarrow \exp \left\{i \int_{0}^{t} d t^{\prime} \alpha\left(t^{\prime}\right)\right\} \psi
$$

where $\alpha(t)$ is a function of time only. Thus, in spite of its apparent simplicity, the effective theory of graphene possesses a truly local gauge invariance, which should be respected by the lattice action. To this end, one introduces temporal link variables

$$
U_{0, \mathbf{n}}=U_{\mathbf{n}} \equiv \exp \left(i \theta_{\mathbf{n}}\right)
$$

where $\theta_{\mathbf{n}}$ is the dimensionless lattice gauge field evaluated at the lattice point $\mathbf{n}=\left(n_{0}, n_{1}, n_{2}, n_{3}\right)$. The spatial link variables

$$
U_{i, \mathbf{n}}=1
$$


are set to unity. It is convenient to express the discretized version of Eq. (2.10) in terms of "plaquette" variables, defined by

$$
U_{\mu v, \mathbf{n}}=U_{\mu, \mathbf{n}} U_{v, \mathbf{n}+\mathbf{e}_{\mu}} U_{\mu, \mathbf{n}+\mathbf{e}_{v}}^{\dagger} U_{v, \mathbf{n}}^{\dagger},
$$

where, in the present case of a pure Coulomb interaction, the only non-trivial components are $U_{0 i}$ and $U_{i 0}$. Those plaquette components then correspond to the discretized formulation of the electric field. The remaining components corresponding to the magnetic field are equal to unity. These statements can be summarized in the expression

$$
U_{\mu v, \mathbf{n}}=\delta_{\mu 0} \delta_{v i} U_{\mathbf{n}} U_{\mathbf{n}+\mathbf{e}_{i}}^{\dagger}+\delta_{v 0} \delta_{\mu i} U_{\mathbf{n}}^{\dagger} U_{\mathbf{n}+\mathbf{e}_{i}}+\delta_{\mu 0} \delta_{v 0}+\delta_{\mu i} \delta_{v j}
$$

In terms of the gauge link variables and plaquettes, the discretized gauge action corresponding to Eq. (2.10) is given by [17]

$$
S_{E}^{g}=\beta \sum_{\mathbf{n}} \sum_{\mu>v}\left[1-\frac{1}{2}\left(U_{\mu v, \mathbf{n}}+U_{\mu v, \mathbf{n}}^{\dagger}\right)\right],
$$

where $\beta=1 / g^{2}$, such that $\beta \rightarrow v_{F} / g^{2}$ when the rescaling of Eq. (2.4) is applied. In Eq. (3.7), the only non-vanishing contributions arise from the terms with $(\mu, v)=(1,0) ;(2,0) ;(3,0) ;(2,1) ;(3,1)$ and $(3,2)$. Eq. (3.7) may be simplified to

$$
S_{E, C}^{g}=\beta \sum_{\mathbf{n}}\left[3-\sum_{i=1}^{3} \Re\left(U_{\mathbf{n}} U_{\mathbf{n}+\mathbf{e}_{i}}^{\dagger}\right)\right],
$$

where $\mathfrak{R}(x)$ denotes the real part of $x$. Eq. (3.8) is referred to as the compact formulation, which has been found to pose problems related to spurious monopole condensation in QED and related theories [18]. On the other hand, the non-compact formulation, which is obtained from Eq. (3.8) by expanding $\Re\left(U_{\mathbf{n}} U_{\mathbf{n}+\mathbf{e}_{i}}^{\dagger}\right)$ to second order in $\theta$,

$$
\Re\left(U_{\mathbf{n}} U_{\mathbf{n}+\mathbf{e}_{i}}^{\dagger}\right)=1-\frac{1}{2}\left(\theta_{\mathbf{n}+\mathbf{e}_{i}}-\theta_{\mathbf{n}}\right)^{2}+\ldots
$$

giving

$$
S_{E, N}^{g}=\frac{\beta}{2} \sum_{\mathbf{n}} \sum_{i=1}^{3}\left(\theta_{\mathbf{n}+\mathbf{e}_{i}}-\theta_{\mathbf{n}}\right)^{2},
$$

is free from such problems $[16,19]$ and allows for a realistic continuum limit.

\subsection{Staggered fermions}

While the discretization of the gauge sector is relatively straightforward, the inclusion of dynamical fermions on the lattice is a notoriously difficult problem. One of the main issues when simulating fermions on the lattice is the so-called doubling problem (for an overview, see Ref. [17], Chapter 4). This problem is related to the chiral invariance of the fermionic sector, and arises due to the appearance of multiple (unwanted) zeros in the inverse propagator. In other words, one is simulating more fermion flavors than expected, the exact number being dependent on the dimensionality of the theory. There exists a number of ways to avoid the doubling problem, but all of them break chiral invariance in one way or the other, a fact encoded in the Nielsen-Ninomiya theorem [20]. 
The solution we have chosen for our simulations of graphene is the "staggered" discretization of Ref. [21]. This choice is advantageous for the study of spontaneous chiral symmetry breaking in graphene, as it yields the correct number of degrees of freedom while (partially) preserving chiral symmetry. The major drawback of staggered fermions is that the full chiral symmetry is restored only in the continuum limit, a fact referred to as "taste symmetry breaking".

In order to discretize the fermionic sector of Eq. (2.2) in a way amenable to computer simulations, a number of choices need to be made. As a first step, fermions are integrated out, and the partition function is written purely in terms of the gauge field, Eq. (2.15). The fermions are then represented exclusively through $\operatorname{det}(D)$. One can then attempt to compute the determinant exactly for a given $\theta$ configuration, which is feasible due to the low dimensionality of the problem, Alternatively, one may rewrite $\operatorname{det}(D)$ in terms of a path integral over complex scalar fields referred to as pseudofermions, as is common in Lattice QCD.

It has been shown in Ref. [22] that for each staggered flavor one recovers, in the continuum limit, two four-component Dirac flavors. Thus, by retaining one staggered flavor, it is possible to have exactly eight continuum fermionic degrees of freedom, which is the correct number for graphene. The action of a single staggered flavor is given by

$$
S_{E}^{f}[\bar{\chi}, \chi, \theta]=-\sum_{\mathbf{n}, \mathbf{m}} \bar{\chi}_{\mathbf{n}} K_{\mathbf{n}, \mathbf{m}}[\theta] \chi_{\mathbf{m}}
$$

where the staggered Dirac operator is

$$
K_{\mathbf{n}, \mathbf{m}}[\theta]=\frac{1}{2}\left(\delta_{\mathbf{n}+\mathbf{e}_{0}, \mathbf{m}} U_{\mathbf{n}}-\delta_{\mathbf{n}-\mathbf{e}_{0}, \mathbf{m}} U_{\mathbf{m}}^{\dagger}\right)+\frac{v_{F}}{2} \sum_{i} \eta_{\mathbf{n}}^{i}\left(\delta_{\mathbf{n}+\mathbf{e}_{i}, \mathbf{m}}-\delta_{\mathbf{n}-\mathbf{e}_{i}, \mathbf{m}}\right)+m_{0} \delta_{\mathbf{n}, \mathbf{m}},
$$

where the phase factors $\eta$ arise from the spin-diagonalization of the Dirac matrices [23]. The operator $K$ thus replaces $D$ in all expressions for the probability, chiral condensate and susceptibility that were derived in the previous sections. As expected from the Nielsen-Ninomiya theorem, the staggered lattice action does not retain the full $\mathrm{U}(4)$ chiral symmetry of the original graphene action at finite lattice spacing. As shown in Ref. [22], only a subgroup $\mathrm{U}(1) \times \mathrm{U}(1)$ remains upon discretization. Spontaneous condensation of $\bar{\chi} \chi$, or equivalently the introduction of a parity invariant mass term, reduces this symmetry to $\mathrm{U}(1)$.

Finally, it should be pointed out that the situation concerning graphene is unusually favorable, in the sense that the staggered formalism fortuitously provides the correct number of fermionic degrees of freedom, as $N_{f}=2$ for graphene monolayers. In general, staggered fermions provide only a compromise solution in the sense that some degree of chiral symmetry is preserved, at the price of retaining some of the doubling originally present in the discretized fermion action. Indeed, if the case of $N_{f}=1$ were to be simulated, it would be necessary to resort to the controversial "rooting" trick [24], whereby the desired number of continuum flavors is restored by taking the appropriate root of the Dirac operator.

\section{Results for $\beta_{c}$}

The results presented in this work for the non-compact gauge action are partly based on the reanalysis of extant data from Ref. [12] and partly on new data on larger lattices. These datasets 

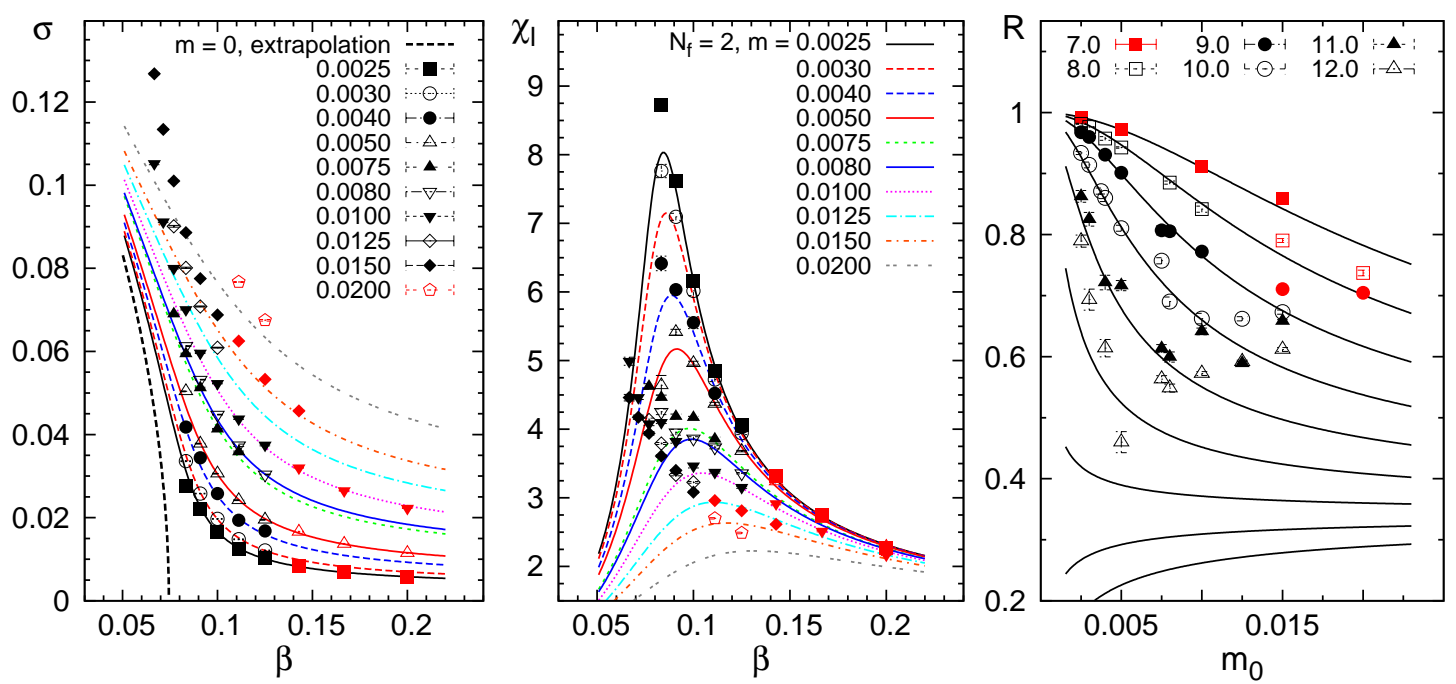

Figure 1: Result of a simultaneous fit to $\sigma$ (left panel), $\chi_{l}$ (middle panel) and $R$ (right panel). Red symbols indicate data for $N_{x}=28$, the remaining ones are for $N_{x}=32$. The datapoints for $R$ are grouped according to $\beta^{-1}$. The fit range has been restricted such that data with $m_{0}>0.005$ and $\beta<0.1$ are excluded, as data at large $m_{0}$ and small $\beta$ have substantial finite volume effects. The optimal parameters are $\beta_{c}=0.0774(2)$ and $\delta=2.93(2)$, with $b=1.0$ fixed. The data satisfy $b=1$ very accurately. The error bars on the datapoints are obtained via the Jackknife method [25].

correspond to $N_{t}=N_{x}=28$ and 32, with the extent of the bulk dimension $N_{b}$ set at either 12 or 32 . We find that finite-size effects are most pronounced as functions of $N_{t}$ and $N_{x}$ at small $\beta$, while the effect of $N_{b}$ on the results is negligible. Our analysis proceeds by first determining the condensate $\sigma$, susceptibility $\chi_{l}$ and $R \equiv \chi_{l} m_{0} / \sigma$ as a function of $\beta$ and $m_{0}$. The second step of the analysis consists of fitting an equation of state (EOS) to $\sigma$ and $\chi_{l}$ in order to obtain estimates of the critical coupling $\beta_{c}$ and the critical exponents. Our EOS is of the form

$$
m_{0} X(\beta)=Y(\beta) \sigma^{b}+\sigma^{\delta},
$$

where the exponent $b \equiv \bar{\beta}-1 / \delta$. This form has been used previously in the context of Lattice QED in $(3+1)$ dimensions [26]. Here, we have referred to the critical exponent using the notation $\bar{\beta}$ to avoid confusion with the inverse coupling $\beta$. The dependence on the critical coupling $\beta_{c}$ enters through the expansions

$$
X(\beta)=X_{0}+X_{1}\left(1-\frac{\beta}{\beta_{c}}\right)+\ldots, \quad Y(\beta)=Y_{1}\left(1-\frac{\beta}{\beta_{c}}\right)+\ldots,
$$

where terms up to $X_{1}$ and $Y_{1}$ have been retained. Higher-order terms were found to have a very small effect on the analysis, and have thus been discarded.

We find that a simultaneous fit to all data for $\sigma, \chi_{l}$ and $R$ yields the most stable results. It is also necessary to carefully consider finite-volume effects and the impact they have on the fit parameters. We have therefore excluded datapoints with $m_{0}>0.005$ and $\beta<0.1$. In this way, we find consistency with mean-field exponents, $\delta=3$ and $b=1$. Including data at lower $\beta$ and larger $m_{0}$ suggests $\delta \sim 2.2$, however such fits have a much higher $\chi^{2}$ and compare unfavorably with the data on $R$. We find that $N_{x}=28$ and $N_{x}=32$ give consistent results using the restricted dataset. 


\section{Experimental situation}

We now turn to the question whether experiments which measure the DC conductivity of suspended graphene provide any evidence for semiconducting behavior which would follow naturally from the excitonic gap scenario. While a full LMC calculation of the conductivity is not yet available, a simplified analysis in terms of a Kubo description of gapped quasiparticles has recently been given in Ref. [27], where the data of Ref. [28] on the suspended graphene devices "S1", "S2" and "S3" were analyzed in terms of the expression $\sigma \equiv \sigma_{q}+\sigma_{b g}$. Here $\sigma_{q}$ is the quasiparticle contribution intrinsic to graphene, while the "background" component $\sigma_{b g}$ is device-dependent.

The Hamiltonian describing Dirac quasiparticles with a gap $\Delta$ and Fermi velocity $v_{F} \simeq c / 300$ is given by $H \equiv \sigma_{1} v_{F} k_{1}+\sigma_{2} v_{F} k_{2}+\sigma_{3} \Delta / 2$, where the $\sigma_{i}$ are Pauli matrices. The contribution $\sigma_{q}$ of the Dirac quasiparticles to the DC conductivity of a graphene monolayer is then

$$
\sigma_{q} \equiv \frac{4 e^{2}}{h} \frac{\pi}{2} \int_{-\infty}^{\infty} d \varepsilon \int_{\Delta / 2}^{\infty} d \xi \xi \mathscr{T}_{\omega}(\xi, \varepsilon) \frac{f\left(\beta \varepsilon-\frac{\beta \omega}{2}-\beta \mu\right)-f\left(\beta \varepsilon+\frac{\beta \omega}{2}-\beta \mu\right)}{\omega}
$$

where $\beta \equiv 1 / k_{B} T$, the Fermi function is given by $f(x)=1 /(1+\exp (x)), \mu$ denotes the chemical potential and the factor of 4 accounts for the spin and valley degrees of freedom. Then

$$
\begin{aligned}
\mathscr{T}_{\omega}(\xi, \varepsilon) & =\frac{\xi^{2}+\Delta^{2} / 4}{\xi^{2}}\left[\delta_{\eta}\left(\xi+\varepsilon+\frac{\omega}{2}\right) \delta_{\eta}\left(\xi-\varepsilon+\frac{\omega}{2}\right)+\delta_{\eta}\left(\xi+\varepsilon-\frac{\omega}{2}\right) \delta_{\eta}\left(\xi-\varepsilon-\frac{\omega}{2}\right)\right] \\
& +\frac{\xi^{2}-\Delta^{2} / 4}{\xi^{2}}\left[\delta_{\eta}\left(\xi-\varepsilon-\frac{\omega}{2}\right) \delta_{\eta}\left(\xi-\varepsilon+\frac{\omega}{2}\right)+\delta_{\eta}\left(\xi+\varepsilon+\frac{\omega}{2}\right) \delta_{\eta}\left(\xi+\varepsilon-\frac{\omega}{2}\right)\right],
\end{aligned}
$$

where $\eta$ is the scattering rate of the quasiparticles, which can be accounted for [29] by broadening the delta functions according to $\pi \delta_{\eta}(x) \equiv \eta /\left(x^{2}+\eta^{2}\right)$. In the DC limit, the integral over $\xi$ in Eq. (5.1) yields

$$
\int_{\Delta / 2}^{\infty} d \xi \xi \mathscr{T}_{0}(\xi, \varepsilon)=\frac{1}{2 \pi}-\frac{\Delta^{2}-4|z|^{2}}{16 \pi \varepsilon \eta} \arg \left(\Delta^{2}-4 z^{2}\right)
$$

where $z \equiv \varepsilon+i \eta$.

The inclusion of the background component $\sigma_{b g}$ is motivated by the fact that the minimal conductivity in graphene samples is non-universal. In suspended graphene, it is much smaller than in graphene samples on a substrate and furthermore strongly sample-dependent. There is also a clear tendency of the minimal conductivity to decrease with increasing sample purity [30]. It is likely that the minimal conductivity in graphene is formed of several components, including the effects of impurities [31], inhomogeneity [32] and effects due to invasive metallic contacts [33]. In our analysis, we have used the phenomenological form

$$
\sigma_{b g} \equiv \sigma_{0} \exp \left[-\left(T_{0} / T\right)^{\alpha}\right]
$$

which allows for the slight empirical $T$-dependence of $\sigma_{b g}$. The empirical data of Ref. [28] is shown in Fig. 2, together with fits in terms of $\sigma_{q}$ and $\sigma_{b g}$. It is noteworthy that the data display a distinct "knee" at $\sim 30 \mathrm{~K}$, which in terms of the present description is interpreted as the temperature below which thermal activation is negligible. Thus, in order to determine $\sigma_{b g}$ in an unbiased fashion, we 

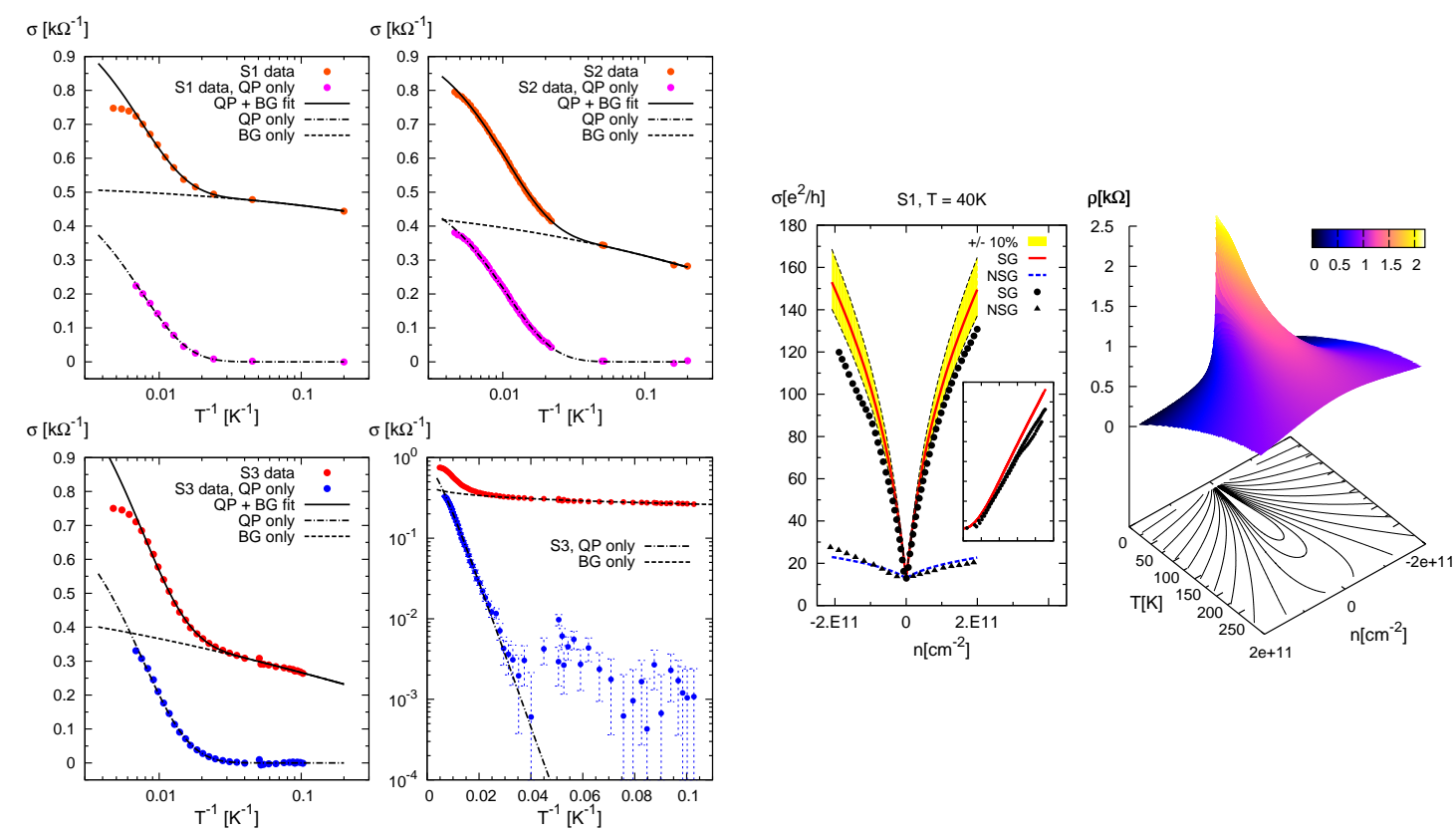

Figure 2: Left panel: quasiparticle (QP) and background (BG) components of $\sigma(n=0, T)$ for the suspended graphene devices S1-S3, as determined in Ref. [27]. The empirical data is reproduced from Ref. [28]. All devices show a "knee" separating thermally activated and background regions. Right panel: $\sigma(n, T)$ determined from a fit to $\sigma(n=0, T)$ and the resistivity $\rho(n, T)$, reproduced from Ref. [27].

first fix $\sigma_{0}$ and $T_{0}$ using data in the extreme low- $T$ region. One may then subtract $\sigma_{b g}$ at all $T$, and determine $\beta \eta$ and $\Delta$ by fitting $\sigma_{q}$ to the resulting dataset. A simultaneous fit of $\sigma_{q}$ and $\sigma_{b g}$ confirms the validity of this procedure. While $\eta(T)$ is a priori unknown, a scenario of constant $\beta \eta$ is strongly favored by the available data in the range $35 \mathrm{~K} \leq T \leq 150 \mathrm{~K}$.

Our findings in Ref. [27] suggest that the suspended graphene devices of Ref. [28] exhibit a thermally activated conductivity $\sigma_{q}$, which is well described by Eq. (5.1) from $T \sim 150 \mathrm{~K}$ down to $T \sim 35 \mathrm{~K}$, where the signal is lost due to limited measurement accuracy. The determined bandgaps are in the range $\Delta \sim 25-40 \mathrm{meV}$, whereas all samples were found to favor $\beta \eta \simeq 0.1$ indicating a scattering rate which increases linearly with $T$. A natural scattering mechanism with such properties is provided by the long-range Coulomb interaction [34] up to logarithmic corrections. Furthermore, this value of $\beta \eta$ is consistent with the high carrier mobilities and long mean free paths reported in Ref. [28], as well as with theoretical estimates of the mean free path due to longrange Coulomb scattering [34]. Specifically, for $T=35-150 \mathrm{~K}$ we find $\eta=3.5-15 \mathrm{~K}$, with corresponding mean free paths of $\hbar v_{F} / \eta \sim 0.5-2.0 \mu \mathrm{m}$. Fits with no gap $(\Delta=0)$, constant $\eta$, or zero background were found to be incompatible with data.

As shown in Fig. 2, these conclusions are consistent with the observed $\sigma(n)$, which depends sensitively on the value of $\beta \eta$ determined from data at $n=0$. Furthermore, the interpretation of the observed $\sigma(n, T)$ as due to thermal activation accounts, in a natural way, for the observed transitional density $n^{*}$ above which $\sigma(T)$ reverts from insulating to metallic. The determined scattering rate $\eta(T)$ is also suggestive of long-range Coulomb scattering, which is consistent with the ultrapure character of the suspended graphene samples of Ref. [28]. 


\section{Conclusions}

We have reviewed the Lattice Gauge Theory approach to the low-energy EFT of graphene, with the aim of introducing this technique to a wider audience and motivate the application of this approach to systems beyond monolayer graphene. Our calculations within this the graphene EFT indicate that it displays a chiral phase transition at a critical coupling of $\beta_{c}=0.0774(2)$, with critical exponents that appear consistent with mean-field theory. Spontaneous chiral symmetry breaking in the graphene EFT would lead to the appearance of a gap in the quasiparticle spectrum, directly linked to the formation of quasiparticle-hole pairs (excitons). It is conceivable that such a transition occurs in suspended graphene, where the strength of the Coulomb interaction attains its maximum value.

In an effort to clarify whether currently available experimental data on suspended graphene provide any evidence for the excitonic scenario, we have reviewed the status of such measurements, which show a definite (though relatively mild) insulating trend at low $T$ in the vicinity of the neutral point. We have presented an interpretation of the observed anomalous temperature dependence in terms of the excitonic gap scenario, and tentatively found that the data may be consistently explained in terms of gapped Dirac quasiparticles $(\Delta \sim 30 \mathrm{meV})$ with the long-range Coulomb interaction as a natural candidate for the dominant scattering mechanism. Further experimental studies of the conductivity at low $T$ in suspended graphene are clearly called for, preferably minimizing the effects of invasive metallic contacts.

Further investigations using the Lattice Gauge Theory approach are in progress, including the renormalization of $v_{F}$ due to the Coulomb interaction, the magnetic catalysis of a semimetalinsulator transition at large external magnetic fields [35, 36], and the critical temperature for exciton condensation in graphene bilayers [37]. The computation of transport properties involves the extraction of spectral functions in Minkowski spacetime from Euclidean time lattice data. Such calculations are also feasible nowadays, as Bayesian analysis coupled with the Maximum Entropy Method has been successfully applied to QCD [38]. Areas of interest include the electrical conductivity and viscosity [39] of the quasiparticles in graphene. Due to the flexibility of the Lattice Monte Carlo and Quantum Monte Carlo methods, further applications are likely to arise in the closely related fields of graphene nanowires, high- $T_{c}$ superconductors and hexagonal optical lattices, to name a few. In summary, the application of Lattice Gauge Theory to condensed matter problems appears poised to develop into a highly fruitful field of study.

\section{References}

[1] K. S. Novoselov et al., Electric field effect in atomically thin carbon films, Science 306, 666 (2004); Two-dimensional atomic crystals, Proc. Natl. Acad. Sci. U.S.A. 102, 10451 (2005); A. K. Geim and K. S. Novoselov, The rise of graphene, Nat. Mat. 6, 183 (2007).

[2] A. H. Castro Neto et al., The electronic properties of graphene, Rev. Mod. Phys. 81, 109 (2009).

[3] J. C. Meyer et al., The structure of suspended graphene sheets, Nature 446, 60 (2007); J. S. Bunch et al., Electromechanical resonators from graphene sheets, Science 315, 490 (2007).

[4] G. W. Semenoff, Condensed matter simulation of a three-dimensional anomaly, Phys. Rev. Lett. 53, 2449 (1984); A. Bostwick et al., Quasiparticle dynamics in graphene, Nat. Phys. 3, 36 (2007).

[5] P. R. Wallace, The band theory of graphite, Phys. Rev. 71, 622 (1947), erratum ibid. 72, 258 (1947). 
[6] S. Reich, J. Maultzsch, C. Thomsen and P. Ordejón, Tight-binding description of graphene, Phys. Rev. B 66, 035412 (2002).

[7] Z. Y. Meng, T. C. Lang, S. Wessel, F. F. Assaad and A. Muramatsu, Quantum spin liquid emerging in two-dimensional correlated Dirac fermions, Nature (London) 464, 847 (2010).

[8] D. T. Son, Quantum critical point in graphene approached in the limit of infinitely strong Coulomb interaction, Phys. Rev. B 75, 235423 (2007).

[9] I. F. Herbut, Interactions and phase transitions on graphene's honeycomb lattice, Phys. Rev. Lett. 97, 146401 (2006).

[10] J. González, F. Guinea and M. A. H. Vozmediano, Non-Fermi liquid behavior of electrons in the half-filled honeycomb lattice (A renormalization group approach); Nucl. Phys. B 424, 595 (1994); Unconventional quasiparticle lifetime in graphite, Phys. Rev. Lett. 77, 3589 (1996); Marginal-Fermi-liquid behavior from two-dimensional Coulomb interaction, Phys. Rev. B 59, 2474(R) (1999).

[11] D. V. Khveshchenko, Massive Dirac fermions in single-layer graphene, J. Phys.: Condens. Matter 21, 075303 (2009).

[12] J. E. Drut and T. A. Lähde, Is graphene in vacuum an insulator? Phys. Rev. Lett. 102, 026802 (2009); Critical exponents of the semimetal-insulator transition in graphene: A Monte Carlo study, Phys. Rev. B 79, 241405(R) (2009).

[13] J. E. Drut and T. A. Lähde, Lattice field theory simulations of graphene, Phys. Rev. B 79, 165425 (2009); A. H. Castro Neto, Pauling's dreams for graphene, Physics (APS) 2, 30 (2009).

[14] S. Hands and C. Strouthos, Quantum critical behavior in a graphenelike model, Phys. Rev. B 78, 165423 (2008); W. Armour, S. Hands and C. Strouthos, Monte Carlo simulation of the semimetal-insulator transition in monolayer graphene, Phys. Rev. B 81, 125105 (2010).

[15] J. B. Kogut, E. Dagotto and A. Kocić, New phase of quantum electrodynamics: A nonperturbative fixed point in four dimensions, Phys. Rev. Lett. 60, 772 (1988); E. Dagotto, J. B. Kogut and A. Kocić, Computer simulation of chiral-symmetry breaking in (2+1)-dimensional QED with $N$ flavors, Phys. Rev. Lett. 62, 1083 (1989); S. J. Hands, J. B. Kogut and C. G. Strouthos, Non-compact QED(3) with $N(f) \geq 2$, Nucl. Phys. B 645, 321 (2002).

[16] M. Göckeler, R. Horsley, E. Laermann, P. E. L. Rakow, G. Schierholz, R. Sommer and U. J. Wiese, QED - A Lattice investigation of the chiral phase transition and the nature of the continuum limit, Nucl. Phys. B 334, 527 (1990); M. Göckeler, R. Horsley, P. Rakow, G. Schierholz and R. Sommer, Scaling laws, renormalization group flow and the continuum limit in non-compact Lattice QED, Nucl. Phys. B 371, 713 (1992).

[17] H. Rothe, Lattice Gauge Theories - An Introduction, $3^{\text {rd }}$ edition, World Scientific, Singapore (2005).

[18] K. Farakos and G. Koutsoumbas, Meson mass calculations in 3D U(1) lattice gauge theory, Phys. Lett. B 178, 260 (1986); J. B. Kogut and C. G. Strouthos, Logarithmic triviality of compact QED coupled to a four-Fermi interaction, Phys. Rev. D 71, 094012 (2005).

[19] A. Kocić, J. B. Kogut and K. C. Wang, Monopole percolation and the universality class of the chiral transition in four flavor noncompact Lattice QED, Nucl. Phys. B 398, 405 (1993).

[20] H. B. Nielsen and M. Ninomiya, Absence Of Neutrinos On A Lattice. 1. Proof By Homotopy Theory, Nucl. Phys. B 185, 20 (1981), erratum ibid. B 195, 541 (1982); Absence Of Neutrinos On A Lattice. 2. Intuitive Topological Proof, Nucl. Phys. B 193, 173 (1981).

[21] J. Kogut, L. Susskind, Hamiltonian formulation of Wilson's lattice gauge theories, Phys. Rev. D 11, 395 (1975); L. Susskind, Lattice fermions, Phys. Rev. D 16, 3031 (1977); H. Kluberg-Stern, A. Morel, O. Napoly and B. Petersson, Flavors of Lagrangian Susskind fermions, Nucl. Phys. B 220, 447 (1983).

[22] C. Burden and A. N. Burkitt, Lattice fermions in odd dimensions, Europhys. Lett. 3, 545 (1987). 
[23] N. Kawamoto and J. Smit, Effective Lagrangian and dynamical symmetry breaking in strongly coupled in strongly coupled Lattice QCD, Nucl. Phys. B, 192, 100 (1981).

[24] M. Creutz, Chrial anomalies and rooted staggered fermions, Phys. Lett. B 649, 230 (2007).

[25] M. C. K. Yang and D. H. Robinson, Understanding and learning science by computer, Series in Computer Science, Vol. 4, World Scientific, Singapore (1986).

[26] M. Göckeler, R. Horsley, V. Linke, P. E. L. Rakow, G. Schierholz and H. Stüben, Seeking the equation of state of non-compact Lattice QED, Nucl. Phys. B 487, 313 (1997); A. Ali Khan, Non-compact Lattice QED with Two Charges: Phase Diagram and Renormalization Group Flow, Phys. Rev. D 53, 6416 (1996).

[27] J. E. Drut, T. A. Lähde and E. Tölö, Signatures of a gap in the conductivity of graphene, [arXiv:1005.5089].

[28] K. I. Bolotin et al., Ultrahigh electron mobility in suspended graphene, Solid State Commun. 146, 351 (2008); Temperature-dependent transport in suspended graphene, Phys. Rev. Lett. 101, 096802 (2008); V. Crespi, Flatland exposed, Physics (APS) 1, 15 (2008).

[29] K. Ziegler, Minimal conductivity of graphene: Nonuniversal values from the Kubo formula, Phys. Rev. B 75, 233407 (2007).

[30] X. Du, I. Skachko, A. Barker and E. Y. Andrei, Approaching ballistic transport in suspended graphene, Nature Nanotechnology 3, 491 (2008).

[31] I. F. Herbut, V. Juričić and O. Vafek, Coulomb interaction, ripples, and the minimal conductivity of graphene, Phys. Rev. Lett. 100, 046403 (2008).

[32] Y. Zhang et al., Origin of spatial charge inhomogeneity in graphene, Nature Phys. 5, 722 (2009).

[33] P. Blake et al., Influence of metal contacts and charge inhomogeneity on transport properties of graphene near the neutrality point, Solid State Comm. 149, 1068 (2009); R. Golizadeh-Mojarad and S. Datta, Effect of contact induced states on minimum conductivity in graphene, Phys. Rev. B 79, 085410 (2009).

[34] L. Fritz, J. Schmalian, M. Müller and S. Sachdev, Quantum critical transport in clean graphene, Phys. Rev. B 78, 085416 (2008).

[35] D. V. Khveshchenko, Magnetic-field-induced insulating behavior in highly oriented pyrolitic graphite, Phys. Rev. Lett. 87, 246802 (2001); D. V. Khveshchenko and H. Leal, Excitonic instability in layered degenerate semimetals, Nucl. Phys. B 687, 323 (2004);

[36] E. V. Gorbar, V. P. Gusynin, V. A. Miransky and I. A. Shovkovy, Magnetic field driven metal-insulator phase transition in planar systems, Phys. Rev. B 66, 045108 (2002).

[37] H. Min, R. Bistritzer, J.-J. Su and A. H. MacDonald, Room-temperature superfluidity in graphene bilayers, Phys. Rev. B 78, 121401 (2008); M. Yu. Kharitonov and K. Efetov, Excitonic condensation in a double-layer graphene system, Semiconductor Science and Technology 25, 034004 (2010).

[38] G. Aarts, C. Allton, J. Foley, S. Hands and S. Kim, Spectral functions at small energies and the electrical conductivity in hot quenched lattice QCD, Phys. Rev. Lett. 99, 022002 (2007).

[39] M. Müller, J. Schmalian and L. Fritz, Graphene: A nearly perfect fluid, Phys. Rev. Lett. 103, 025301 (2009). 